\title{
Finding the Planck Length Independent of Newton's Gravitational Constant and the Planck Constant The Compton Clock Model of Matter
}

\author{
Espen Gaarder Haug \\ Norwegian University of Life Sciences \\ September 19, 2018
}

\begin{abstract}
In modern physics, it is assumed that the Planck length is a derived constant from Newton's gravitational constant, the Planck constant and the speed of light, $l_{p}=\sqrt{\frac{G \hbar}{c^{3}}}$. This was first discovered by Max Planck in 1899. We suggest a way to find the Planck length independent of any knowledge of Newton's gravitational constant or the Planck constant, but still dependent on the speed of light (directly or indirectly).

Key words: Planck length, Planck constant, Newton's gravitational constant, Compton frequency, Schwarzschild radius.
\end{abstract}

\section{INTRODUCTION}

In 1899, Max Planck [1, 2] introduced what he called the 'natural units': the Planck mass, the Planck length, the Planck time, and the Planck energy. He derived these units using dimensional analysis, assuming that Newton's gravitational constant, the Planck constant, and the speed of light were the most important universal constants. The Max Planck formula for the Planck length is given by

$$
l_{p}=\sqrt{\frac{G \hbar}{c^{3}}}
$$

In other words, it seems that we need to know Newton's gravitational constant $G$ and the Planck constant $\hbar$ and the speed of light $c$ to find the Planck length. It has therefore been assumed that the Planck length is a derived constant and that Newton's gravitational constant is a more fundamental constant, a view we will challenge here.

\section{PLANCK LENGTH INDEPENDENT OF $G$ AND $\hbar$}

First, find the reduced Compton wavelength of an electron by Compton scattering. The reduced Compton frequency is then given by

$$
f_{C}=\frac{c}{\bar{\lambda}_{e}}
$$

where $f_{C}$ stands for the reduced Compton frequency, and $\bar{\lambda}_{e}$ is the reduced Compton wavelength of the electron.

Further, the Cyclotron frequency is linearly proportional to the reduced Compton frequency. Conducting a cyclotron experiment, one can find the reduced Compton frequency ratio between the proton and the electron. For example, [3] measured it to be about

$$
\frac{\frac{c}{\lambda_{P}}}{\frac{c}{\lambda_{e}}}=\frac{f_{C, P}}{f_{C, e}}=1836.152470(76)
$$

In fact, [3] measured the Proton electron mass ratio this way, but the reduced Compton frequency is only a deeper aspect of mass, that has recently also been more or less confirmed by experimental research. Theoretically, it is no surprise that $\frac{f_{C, P}}{f_{C, e}}=\frac{m_{P}}{m_{e}}$. We simply claim that a simpler and deeper way to express mass is through the reduced Compton frequency in matter; see [4].

Next, from the Schwarzschild metric [5, 6] solution of the Einstein field equation [7], we have the well-known formula for the Schwarzschild radius

$$
r_{s}=\frac{2 G M}{c^{2}}
$$

Haug [4] recently pointed out that the Schwarzschild radius is also equal to twice the reduced Compton frequency of the gravity object over a time period of one Planck second, multiplied by the Planck length. That is, we must have

$$
\frac{r_{s}}{2}=\frac{G M}{c^{2}}=f_{C} t_{p} l_{p}=\frac{c}{\bar{\lambda}} \frac{l_{p}}{c} l_{p}=\frac{l_{p}^{2}}{\bar{\lambda}}
$$

This means that the Schwarzschild radius contains the Planck length. Still, is there a way to extract this without knowing the mass and Newton's gravitational constant? Haug [8] recently showed that the Schwarzschild radius can be measured in a series of ways with no knowledge of the mass size or Newton's gravitational constant. For example, the Schwarzschild radius of the earth can be found from the gravitational acceleration field, the speed of light and the radius of the earth 


$$
\frac{r_{s}}{2}=\frac{g R^{2}}{c^{2}}
$$

where $g$ is the gravitational acceleration and $R$ is the radius of the gravity object. For smaller size objects, we can find the Schwarzschild radius using a Cavendish apparatus; it is given by

$$
r_{s}=\frac{L 4 \pi^{2} R^{2} \theta}{c^{2} T^{2}}
$$

where $L$ is the distance between the small balls and $R$ is the distance, center to center, between the small and large ball. $T$ is the natural resonant oscillation period of a torsion balance, $\theta$ is the deflection angle of the balance. This is somewhat different than the known use of a Cavendish apparatus used to find the weight of the Earth or to find $G$, where one needs to know the mass size (weight) of the lead balls. Again, we do not need to know the mass size in the normal sense nor do we need to know anything about Newton's gravitational constant to find the Schwarzschild radius.

So, in practice, take a clump of matter, divide it in two and make two "identical" balls of it. Place the two balls in the Cavendish apparatus. In addition, one needs two much smaller balls. By measuring $T, L, R$ and $\theta$, we know the Schwarzschild radius of the large balls in the apparatus. Assume that we measure a Schwarzschild radius of $7.43 \times 10^{-28}$ meters.

Next, we find the reduced Compton frequency of an electron: it is

$$
f_{C, e}=\frac{c}{\bar{\lambda}_{e}} \approx 7.76 \times 10^{20}
$$

Next, we use the cyclotron to find the reduced Compton frequency of a Proton

$$
f_{C, P}=\frac{c}{\bar{\lambda}_{e}} \times 1836.15247 \approx 1.42549 \times 10^{24}
$$

Next, we break the large balls up into pieces and count the number of protons in it. Or, alternatively, we can pack a known amount of atoms together in a ball, and keep track of the number of protons. This is, in theory at least, possible. Assume that we count approximately $5.97864 \times 10^{26}$ protons in the mass, and we next divide it into two. The reduced Compton frequency is additive for a larger mass, so based on the proton count the reduced Compton frequency in each of the large balls must be approximately

$$
\begin{aligned}
f_{C} & =1.42549 \times 10^{24} \times \frac{1}{2} \times 5.97864 \times 10^{26} \\
& \approx \frac{1}{2} \times 8.52249 \times 10^{50}
\end{aligned}
$$

Remember that the Schwarzschild radius that we measured was $7.43 \times 10^{-28} \mathrm{~m}$. To find the Planck length, we can use the following formula

$$
l_{p}=\sqrt{\frac{1}{2} \frac{r_{s}}{f_{C}} c}
$$

This gives us

$$
l_{p}=\sqrt{\frac{1}{2} \frac{r_{s}}{f_{C}}} c \approx \sqrt{\frac{7.43 \times 10^{-28}}{8.52249 \times 10^{50}}} c \approx 1.616 \times 10^{-35} \mathrm{~m}
$$

This also means that the Planck length is always the square root of half the Schwarzschild radius times the reduced Compton wavelength of the mass

$$
l_{p}=\sqrt{\frac{1}{2} r_{s} \bar{\lambda}}
$$

The whole method requires no knowledge of traditional mass size measures, which also means no knowledge of the Planck constant $\hbar$, and no knowledge of Newton's gravitational constant $G$.

\section{THE PLANCK TIME AND THE PLANCK MASS}

After we have found the Planck length, completely independent of the Planck constant and the gravitational constant, we can naturally easily find the Planck time as it is simply the Planck length divided by the speed of light. However, the Planck mass and all masses at the deepest level can be described as Compton frequency per Planck second. This means that the Planck mass in this view is

$$
\hat{m}_{p}=f_{C} t_{p}=\frac{c}{l_{p}} \frac{l_{p}}{c}=1
$$

That is, the Planck mass is one in terms of frequency per Planck second. That mass is related to Compton time has also been supported in recent experimental research. Dolce and Perali [9] conclude that "the rest-mass of a particle is associated to a rest periodicity known as Compton periodicity". Additionally, [10] concluded that "This directly demonstrates the connection between time and mass". In our view, the reduced Compton frequency hidden inside matter is the key to really understand how to extract the Planck length independent of the Planck constant and to understand mass and gravity from a deeper perspective. 


\section{AN ALTERNATIVE WAY THAT IS INDEPENDENT OF $G$ BUT DEPENDENT ON KNOWING $\hbar$}

At a deeper level then, the Planck constant must somehow contain an embedded reduced Compton frequency for one $\mathrm{kg}$. The reduced Compton wavelength for $1 \mathrm{~kg}$ is

$$
\bar{\lambda}=\frac{\hbar}{1 \mathrm{~kg} \times c}=\frac{\hbar}{c}
$$

The reduced Compton frequency of $1 \mathrm{~kg}$ is therefore

$$
f_{C, 1 \mathrm{~kg}}=\frac{c}{\frac{\hbar}{1 \mathrm{~kg} \times c}}=\frac{c^{2} \times 1 \mathrm{~kg}}{\hbar} \approx 8.52 \times 10^{50}
$$

That is, $\frac{c^{2}}{\hbar}$ is simply the frequency of $1 \mathrm{~kg}$. Next, let us make two balls with weight $1 \mathrm{~kg}$ and also two much smaller balls to use in a Cavendish apparatus. Next, we measure the Schwarzschild radius using the apparatus. There is no need to know the Planck constant or the weight of the balls to find the Schwarzschild radius. It is when we are going to extract the Planck length from the Schwarzschild radius that we, in this method, will utilize the Planck constant. This is because the Planck constant is needed to extract the reduced Compton frequency in the matter when working with $\mathrm{kg}$. In the section above, we had to count the number of protons, but if working from $\mathrm{kg}$ instead we can use the Planck constant to extract the reduced Compton frequency. The Planck length from the measured Schwarzschild radius in $1 \mathrm{~kg}$ is simply equal to

$$
\begin{aligned}
l_{p} & =\sqrt{\frac{1}{2} \frac{r_{s, 1 \mathrm{~kg}}}{f_{C}} c} \\
& \approx \sqrt{\frac{7.43 \times 10^{-28}}{\frac{c^{2}}{\hbar}}} \\
& \approx \sqrt{\frac{7.43 \times 10^{-28}}{8.522 \times 10^{50}}} \approx 1.616 \times 10^{-35} \mathrm{~m}
\end{aligned}
$$

$\mathrm{S}$

It is also worth mentioning that the Planck frequency is given by the reduced Compton frequency of the matter in question multiplied by the Planck length divided by half the Schwarzschild radius

$$
f_{C, p}=\frac{f_{C} l_{p}}{\frac{1}{2} r_{s}}=\frac{c}{l_{p}}
$$

\section{RELATION TO MASS AS KG}

In the above sections, we have shown that we can find the Planck length independent of any knowledge of Newton's gravitational constant. We have also claimed that the Planck mass could simply be described by its Compton frequency.

It is only if we want to convert the Planck mass frequency per Planck second into $k g$ that we really need the Planck constant. Any mass in $\mathrm{kg}$ can be found if we know the reduced Compton wavelength of the mass in question

$$
m=\frac{\hbar}{\bar{\lambda}} \frac{1}{c}
$$

We have found the Planck length independent of $G$ and $\hbar$, but need the Planck constant to find the Planck mass in terms of $\mathrm{kg}$. This gives

$$
m_{p}=\frac{\hbar}{l_{p}} \frac{1}{c}=2.1765 \times 10^{-8} \mathrm{~kg}
$$

The reduced Compton frequency of $1 \mathrm{~kg}$ is, as we claimed earlier in this paper,

$$
f_{C, 1 \mathrm{~kg}}=\frac{c}{\frac{\hbar}{1 \mathrm{~kg} \times c}}=\frac{c^{2}}{\hbar} \approx 8.52 \times 10^{50}
$$

This means the Planck constant is equal to

$$
\hbar=\frac{c^{2}}{8.52 \times 10^{50}} \approx 1.0546 \times 10^{-34}
$$

Now let us replace this into the mass equation

$$
m=\frac{\hbar}{\bar{\lambda}} \frac{1}{c}=\frac{\frac{c^{2}}{8.52 \times 10^{50}}}{\bar{\lambda}} \frac{1}{c}=\frac{\frac{c}{\lambda}}{8.52 \times 10^{50}}=\frac{f_{C}}{f_{C, 1 \mathrm{~kg}}}
$$

That is, any mass in $\mathrm{kg}$ form is simply the reduced Compton frequency of the mass in question divided by the reduced Compton frequency of $1 \mathrm{~kg}$. So, the Planck constant is simply needed to make our mass unit into a $\mathrm{kg}$ measure/definition of mass. An alternative would be to describe mass as the reduced Compton frequency over one Planck second. This means that all rest-masses can now be simply described as

$$
\hat{m}=f_{C} t_{p}=\frac{c}{\bar{\lambda}} \frac{l_{p}}{\bar{\lambda}}=\frac{l_{p}}{\bar{\lambda}}
$$

Any mass smaller than a Planck mass will then somehow be probabilistic as it means a frequency of less than one per Planck second. This could explain why quantum mechanics is necessary for the quantum world, but not in the macroscopic world; see [4] for more details along these lines. 


\section{WHY NEWTON'S GRAVITATIONAL CONSTANT IS A COMPOSITE CONSTANT}

Haug has, in a series of papers [11-13], suggested that Newton's gravitational constant is a composite constant of the form

$$
G=\frac{l_{p}^{2} c^{3}}{\hbar}
$$

which is basically the same as the McCulloch [14] constant $\frac{\hbar c}{m_{p}^{2}}$ since $m_{p}=\frac{\hbar}{l_{p}} \frac{1}{c}$. Here, we will list some reasons that seem to support the fact that Newton's gravitational constant is a Composite constant rather than a deep fundamental constant.

- Already, when looking at the output units of Newton's gravitational constant $\mathrm{m}^{3} \cdot \mathrm{kg}^{-1} \cdot \mathrm{s}^{-2}$, one should become suspicious that this must be a composite constant. It is highly unlikely that the universe invented something fundamental that has some complex units. Much more likely is that the Planck length is something very fundamental. Everyone can intuitively understand, to a certain extent, what a length is. One can also, to a large degree, understand what the speed of light is: it is how far the light travels in a given time period. The Planck length is the shortest possible reduced Compton wavelength.

- The Planck length can be found completely independent of any knowledge of Newton's gravitational constant; it can also be found independent of the Planck constant, as shown in this paper. The Planck constant in the Max Planck formula, in our view, is actually needed in that formula to cancel out the Planck constant embedded in Newton's gravitational constant.
- By re-formulating $G$ as a composite of the form $G=\frac{l_{p}^{2} c^{3}}{\hbar}$, a long series of Planck units is dramatically simplified and becomes more logical; see [11]. The Planck time described as $t_{p}=\sqrt{\frac{G \hbar}{c^{5}}}$ gives minimal intuition. We may ask, what is the logical meaning of $c^{5}$ and what is the deep logic behind the gravitational constant? First, when replacing $G$ with its composite form, we see that the Planck time is simply $t_{p}=\frac{l_{p}}{c}$, so the time it takes for light to travel the Planck length is naturally known. All the Planck units seem mystical when calculated from Newton's gravitational constant and the Planck constant. Only when we have an output in $\mathrm{kg}$, do we need the Planck constant.

We do not question that Newton's gravitational constant is a universal constant, but it is, in our view, clearly a composite constant that consists of more fundamental entities.

\section{CONCLUSION}

We have, for the first time, shown how the Planck length can be measured without any knowledge of Newton's gravitational constant and the Planck constant. It seems that the Compton frequency of matter is what is important for understanding mass at a deeper level. Further, the Schwarzschild radius is the reduced Compton frequency over the Planck time period multiplied by the Planck length. All we need to extract the Planck length is to know the reduced Compton frequency of matter and the Schwarzschild radius. The Schwarzschild radius also seems to play a central role for gravity. We claim that it is the reduced Compton frequency in matter, over the shortest time interval possible, multiplied by the shortest length possible. This view on the Schwarzschild radius seems to possibly be key to understanding gravity at a deeper level. This should encourage further investigation, theoretically and experimentally.
[1] Max Planck. Naturlische Maasseinheiten. Der Königlich Preussischen Akademie Der Wissenschaften, p . 479., 1899.

[2] Max Planck. Vorlesungen über die Theorie der Wärmestrahlung. Leipzig: J.A. Barth, p. 163, see also the English translation "The Theory of Radiation" (1959) Dover, 1906.

[3] R.S. Van Dyck, F.L. Moore, D.L. Farnham, and P.B. Schwinberg. New measurement of the proton-electron mass ratio. International Journal of Mass Spectrometry and Ion Processes, 66.

[4] E. G. Haug. Revisiting the derivation of Heisenberg's uncertainty principle: The collapse of uncertainty at the Planck scale. preprints.org, 2018.

[5] K. Schwarzschild. Über das gravitationsfeld eines massenpunktes nach der einsteinschen theorie. Sitzungsberichte der Deutschen Akademie der Wissenschaften zu
Berlin, Klasse fur Mathematik, Physik, und Technik, page 189, 1916.

[6] K. Schwarzschild. über das gravitationsfeld einer kugel aus inkompressibler flussigkeit nach der einsteinschen theorie. Sitzungsberichte der Deutschen Akademie der Wissenschaften zu Berlin, Klasse fur Mathematik, Physik, und Technik, page 424, 1916.

[7] Albert Einstein. Näherungsweise integration der feldgleichungen der gravitation. Sitzungsberichte der Königlich Preussischen Akademie der Wissenschaften Berlin, 1916.

[8] E. G. Haug. Gravity without Newton's gravitational constant and no knowledge of mass size. preprints.org, 2018.

[9] D. Dolce and Perali. On the Compton clock and the undulatory nature of particle mass in graphene systems. The European Physical Journal Plus, 130(41), 2015.

[10] S. Lan, P. Kuan, B. Estey, D. English, J. M. Brown, M. A. Hohensee, and Müller. A clock directly linking 
time to a particle's mass. Science, 339, 2013.

[11] E. G. Haug. The gravitational constant and the Planck units. a deeper understanding of the quantum realm. www.viXra.org 1603.0207, 2016.

[12] E. G. Haug. Planck quantization of newton and einstein gravitation. International Journal of Astronomy and As- trophysics, 6(2), 2016.

[13] E. G. Haug. Newton and Einstein's gravity in a new perspective for Planck masses and smaller sized objects. International Journal of Astronomy and Astrophysics, 2018.

[14] M. E. McCulloch. Gravity from the uncertainty principle. Astrophysics and Space Science, 349(2), 2014. 